Images du travail, travail des images

1 | 2016

Quand les groupes professionnels se mettent en images

\title{
Les professionnels de l'image à l'écran : les cinéastes animaliers se mettent en scène
}

Maxence Lamoureux

\section{OpenEdition}

\section{Journals}

Édition électronique

URL : http://journals.openedition.org/itti/1302

DOI : 10.4000/itti. 1302

\section{Éditeur}

Université de Poitiers

Référence électronique

Maxence Lamoureux, « Les professionnels de l'image à l'écran : les cinéastes animaliers se mettent en scène », Images du travail, travail des images [En ligne], 1 | 2016, mis en ligne le 01 février 2016, consulté le 14 avril 2021. URL : http://journals.openedition.org/itti/1302 ; DOI : https://doi.org/10.4000/itti.1302

Ce document a été généré automatiquement le 14 avril 2021

Images du travail, travail des images 


\title{
Les professionnels de l'image à l'écran : les cinéastes animaliers se mettent en scène
}

\author{
Maxence Lamoureux
}

Dans une thèse consacrée aux réalisateurs documentaristes français, Mathias Blanc ${ }^{1}$, note que les années 1990 marquent une phase d'institutionnalisation du documentaire, avec le développement des fonds de soutiens, des reconnaissances parmi les pouvoirs publics et l'émergence des organisations professionnelles. En parallèle, on assiste à une théorisation esthétique de l'activité et des œuvres. En élaborant des discours sur leur pratique, en théorisant le documentaire de création, les cinéastes contribuent à façonner les contours de leur profession. Les propos des cinéastes ne passent pas uniquement par la voie de manifestes ou de lettres ouvertes, aussi utilisent-ils leur média privilégié comme un outil de communication. Ainsi nous pouvons analyser ce qui participe à leur discours dans les images qu'ils produisent de leur activité. L'image estelle un outil qui leur permet de montrer la réalité de leur métier ou bien qui leur permet de construire une vision plus orientée de leur profession? De notre côté, pouvons-nous utiliser l'analyse de l'image produite par des professionnels pour répondre en partie à une recherche sociologique consacrée à un groupe de cinéastes ?

2 Un exemple de spécialisation technique au sein de la production audiovisuelle nous permettra d'analyser comment un petit groupe de travailleurs de l'image élabore l'identité de son activité dans le cadre d'un questionnement sur la construction en cours d'un groupe professionnel. Dans cette optique, nous nous intéresserons aux cinéastes animaliers comme catégorie de professionnels de l'image très restreinte et facilement identifiable par sa production. Le genre animalier, dans lequel nous regroupons des films documentaires et de fiction, recouvre des réalités esthétiques différentes et des contextes de productions variés. Le film animalier, essentiellement documentaire, a suivi la même évolution avec le développement important de la production télévisuelle. Ce marché a permis la spécialisation de quelques cinéastes et la reconnaissance d'un savoir-faire spécifique à la captation de l'image de l'animal 
sauvage ${ }^{2}$. On retrouve des indices de l'institutionnalisation à travers la création en 2004 d'un diplôme "réalisation de documentaire animalier " par l'Université de Poitiers et de l'institut francophone de formation au cinéma animalier (IFFCAM). Cependant la diversité des contextes de production, le travail audiovisuel découpé par projets successifs et la dispersion géographique des professionnels ne favorisent pas les échanges entre cinéastes animaliers. De plus, l'origine sociale et les profils de formation des cinéastes ont évolué dans le temps. Alors que les cinéastes animaliers se définissaient dans les années 1980 comme des autodidactes, techniciens de terrain et naturalistes, progressivement sont apparues des figures de scientifiques ayant l'expérience et la légitimité de porter un discours sur le monde sauvage. Le développement du genre à la télévision a ensuite favorisé l'apparition de réalisateurs de formation audiovisuelle ou journalistique, des conteurs d'histoires et fabricants d'images dont l'expérience de la nature est moindre. « Cinéaste animalier » ne sont plus des termes qui définissent celui dont l'activité consiste à aller filmer dans la nature des animaux sauvages et à transformer ces images en œuvres audiovisuelles. Désormais, il s'agit avant tout de l'expérience sociale de la médiation filmique de la nature. L'émergence du groupe professionnel des cinéastes animaliers se fait à travers le partage de cette expérience sociale. ${ }^{3}$ Les images des cinéastes animaliers au travail, qu'ils produisent eux-mêmes, participent à la création de cette identité professionnelle commune.

3 Il existe deux sources d'images des cinéastes dans leurs activités. Les making-of, qui, réalisés en parallèle des films principaux, se sont développés depuis la vente des films en DVD. Le développement d'Internet a permis aussi de communiquer largement autour d'un film avant même le tournage. Par exemple, le film de Luc Jacquet, Il était une forêt (2013) a disposé de son site internet avant même le début du tournage. Les premiers films issus des repérages furent mis en ligne très tôt lors de la pré-production $\mathrm{du}$ film. Ensuite, durant et après le tournage étaient postés régulièrement, en direct depuis le Pérou et le Gabon, des vidéos présentant quelques petites histoires de l'équipe ${ }^{4}$. Au-delà des making-of, certains films eux-mêmes proposent des images des cinéastes en activité. La mise en scène des réalisateurs ou des chefs-opérateurs est un outil de construction de récit de plus en plus employé. Ils témoignent devant la caméra de l'aventure qu'ils vivent tout en effectuant leur travail.

4 Au-delà de l'analyse esthétique et narrative par laquelle il est nécessaire de passer afin de mettre en lumière les éléments de construction de l'image des cinéastes, il faut se demander : Comment les cinéastes animaliers se représentent-ils dans leur activité et dans leur vie extraprofessionnelle? Quelles sont les intentions derrière le choix des extraits assemblés en film?

\section{Le développement des images du cinéaste au travail}

5 Dans les DVD, les making-of sont des arguments de vente essentiels. D'après une étude du Centre National de la Cinématographie et de l'image animée ${ }^{5}$, il s'agit du deuxième bonus le plus présent dans toutes les éditions DVD (plus d'un DVD sur deux en contient un) après les bandes-annonces. Les bandes-annonces étant des produits de marketing qui existaient bien avant l'invention du DVD, les making-of constituent la réalisation filmique la plus importante créée par l'industrie du film avec le développement de ce 
nouveau support de diffusion. Cette même étude du CNC confirme que le making-of est le 'bonus' le plus attendu par le public, surtout lorsqu'il s'agit de film d'auteur (pour un public que cette étude qualifie de "cultivé») ou de films qui font appel à des techniques particulières comme des effets spéciaux ou du maquillage (pour un public dit « populaire »). Or les films animaliers portent en eux ces deux aspects pour ces deux publics. Le premier public, qui connaît la nature et s'intéresse aux sujets animaliers, est friand d'informations complémentaires sur les sujets abordés. Le deuxième public, plus profane en matière de connaissances en histoire naturelle, s'intéresse au voyage effectué par l'équipe de tournage ainsi qu'aux astuces utilisées pour filmer les animaux.

6 A côté de ces making-of la présence de l'humain dans le film animalier est un élément qui se développe fortement depuis une dizaine d'années, notamment sous l'impulsion des chaînes de télévision. La présence d'un homme, scientifique, aventurier ou le cinéaste lui-même permettrait au spectateur de s'identifier à un personnage et de suivre ses aventures. Au-delà de l'intérêt dramaturgique, il existe aussi un intérêt économique. Le documentaire sur la nature, notamment le genre animalier, possède un coût de production élevé, dû aux temps de développement, de repérage et de tournage nécessairement plus longs lorsqu'il s'agit de filmer des animaux sauvages. Un film documentaire est moins coûteux lorsqu'il comporte davantage d'humains: les interviews et les mises en situation sont plus facilement réalisées. De plus, depuis l'arrivée de la technologie numérique, il est plus facile pour les cinéastes de se filmer eux-mêmes. Auparavant, la pellicule, très couteuse, avec des durées d'enregistrement limitées à quelques minutes, ne favorisait pas la prise de vues de soi. Désormais les caméras vidéo numériques permettent de filmer durant plusieurs minutes et de réenregistrer sur le même support. Un cinéaste animalier, le plus souvent seul sur le terrain, peut beaucoup plus facilement laisser tourner la caméra à un endroit et s'éloigner d'elle pour passer dans le champ.

7 Les films purement «nature ", qui se passe d'humains pour commenter et informer le spectateur, sont rares, plus chers et exigeants, ainsi que plus difficiles d'accès pour un public non averti ${ }^{6}$. Ajouter de l'humain, c'est donc permettre la projection du spectateur au sein du film, c'est le prendre par la main pour aiguiser son intérêt. La voix-off est une solution quasi-systématique dans les productions télévisuelles, mais d'autres solutions existent. Quelques films animaliers de cinéma utilisent des humains comme des personnages de fiction (Le Renard et l'enfant (Luc Jacquet, 2007), Océans (Jacques Perrin et Jacques Cluzaud, 2010), Génésis (Claude Nuridsanny, Marie Pérennou, 2002), La Clé des champs (Claude Nuridsanny, Marie Perennou, 2011)). Cependant l'intégration de scènes de fiction au sein du documentaire pose question. Notamment dans les productions télévisuelles animalières catégorisées en tant que « documentaire » par les diffuseurs et le $\mathrm{CNC}^{7}$. Dans ces productions, l'un des humains le plus facile à intégrer à un récit documentaire, sans qu'il ne devienne un récit de fiction, est le travailleur de l'image. Cette tendance se retrouve également dans des films autoproduits, comme le sont les films des étudiants de l'Institut Francophone de Formation au Film Animalier de Ménigoute. Se filmer en train de filmer, en train de suivre la piste d'un animal, ou tout simplement en train de se promener dans la nature, est une figure narrative très présente dans les productions des étudiants ${ }^{8}$. Ils évitent ainsi d'avoir recours à des acteurs et à des mises en scène de fiction complexes pour lesquelles ils ne sont pas formés. Dans ces différents cas, le film animalier devient la quête d'un homme. 


\section{L'intérêt dramaturgique de la mise en scène du cinéaste animalier}

8 Dans son film, Vertige d'une rencontre (2010), le réalisateur Jean-Michel Bertrand choisit d'établir une distinction visuelle très forte entre les scènes dans lesquelles il est présent et les scènes de nature. En post-production, le réalisateur a désaturé ${ }^{9}$ les couleurs des séquences dans lesquelles il apparaît. Ces mêmes séquences sont généralement filmée avec des caméras peu stables : à l'épaule ou en vues subjectives via une petite caméra numérique. Les séquences nature sont quant à elles en couleurs vives et naturelles et toujours filmées sur pied.

\section{Images extraites du film Vertige d'une rencontre.}
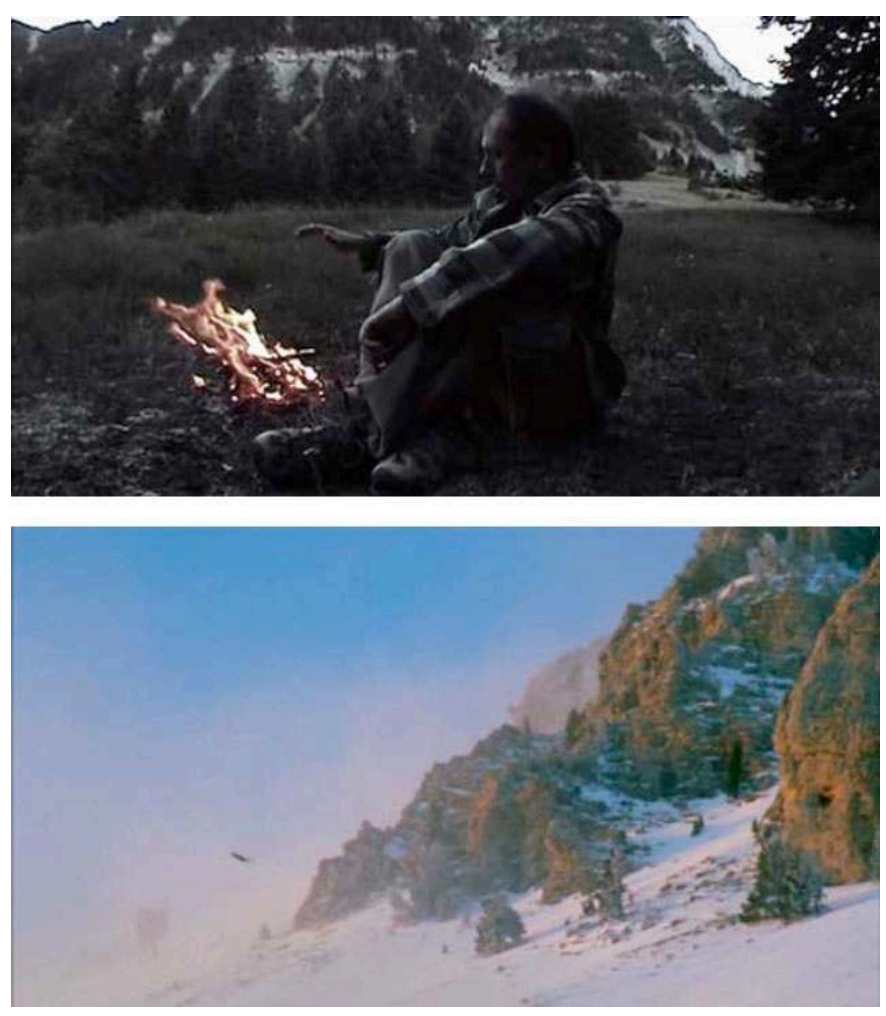

Lorsque le film évoque l'aventure du réalisateur, les images sont désaturées en couleurs (image de gauche), lorsqu'il parle de la nature sauvage les couleurs sont naturelles (image de droite).

Comment justifier cette distinction alors que dans certaines séquences Jean-Michel Bertrand croise des animaux sauvages mais conserve la désaturation? Ailleurs, d'autres plans nature, pourtant issus d'observations du cinéaste conservent leurs couleurs. Le choix de mettre deux voix-off différentes, celle du réalisateur et celle de Charles Berling, une pour chaque type de séquence, nous donne des indices. Deux films sont en réalité mélangés. Le premier est un film animalier, avec la voix-off d'un acteur connu, et dont le sujet ne porte que sur le monde sauvage. Le deuxième est le making-of de ce film : le réalisateur nous présente le tournage comme une aventure. Le film de JeanMichel Bertrand apparaît donc comme un étonnant assemblage du film principal et du making-of. Les deux se répondent et se complètent pour créer une dynamique et un récit unique. Le film animalier principal, sans l'apport des séquences de making-of, 
apparaît classique dans sa forme documentaire, dans son déroulement. Les séquences making-of, très construites et découpées, viennent apporter un autre style d'écriture, plus fictionnel.

Au début du film Voyage au bout de l'Hiver, d'Anne et Erik Lapied (2010), le cinéaste est filmé en train d'escalader une paroi très raide. Le montage met en parallèle des bouquetins dans des milieux similaires. En voix-off, Erik nous raconte sa découverte du milieu alpin au plus jeune âge et la naissance de sa passion. Il explique comment il a appris la montagne et pourquoi il partage la même passion avec sa femme. Il décrit les risques qu'il prend pour accomplir son métier et les connaissances qu'il doit avoir du milieu alpin afin de s'y mouvoir et d'y travailler. A l'image les deux premiers plans sont extrêmement parlants. Le premier montre Erik grimpant dans l'ombre, en contre-jour par rapport au décor des montagnes lointaines. Dans le deuxième, un chamois se découpe dans l'ombre de la montagne, filmé en contre-plongée et sous exposé par rapport à la paroi rocheuse derrière lui. L'animal de son point de vue dominant semble regarder ce grimpeur venu d'en bas. Ces deux images placent leurs sujets dans l'ombre : deux silhouettes associées devant l'immensité de la montagne. L'homme essaye de se fondre dans le paysage, pour un instant il ressemble au bouquetin.

\section{Images extraites du film Voyage au bout de l'hiver.}
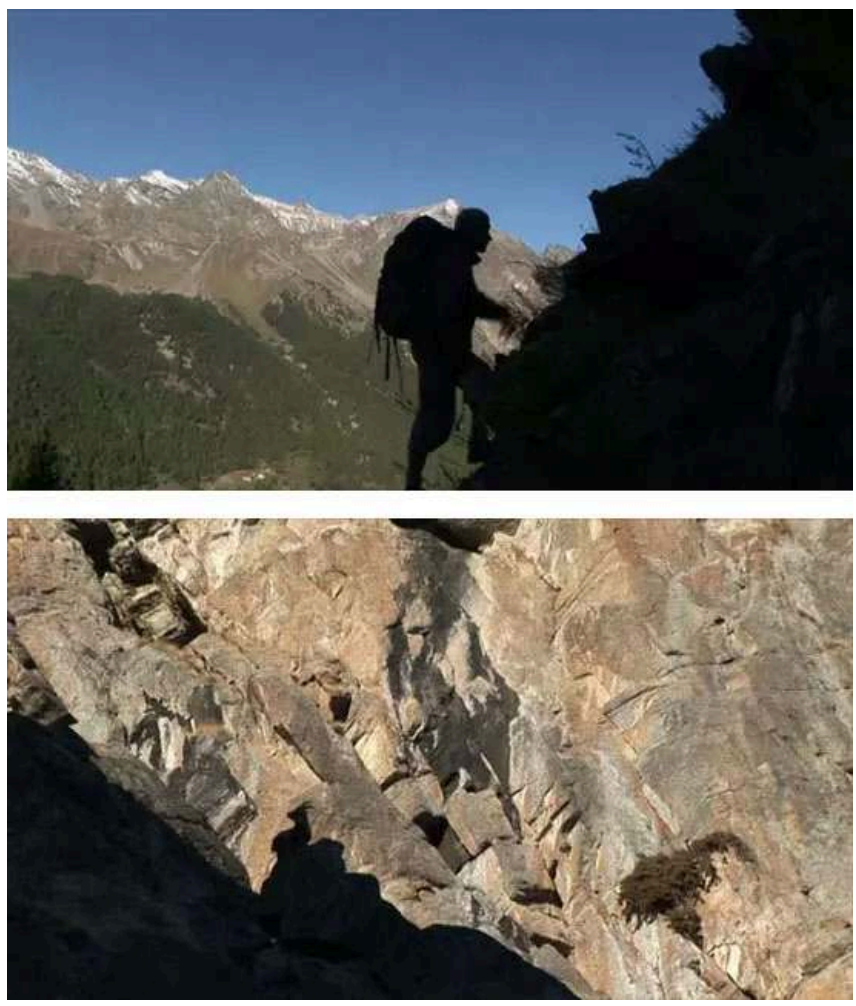

Le premier plan montre le cinéaste en silhouette en train d'escalader une paroi rocheuse sur fond de montagne. Le plan suivant montre l'animal sauvage, un bouquetin, en silhouette sur fond de montagne.

11 Cette séquence est extrêmement découpée. L'escalade du cinéaste est montrée sous plusieurs angles et plusieurs rapports de taille. Le montage très narratif alterne plans larges, plans moyens et gros plans. Parallèlement sont insérées quelques images des bouquetins qui continuent à observer et à faire leur vie non loin de là. Ce découpage narratif pourraient être reçu par le spectateur comme celui d'un film de fiction si la 
voix-off et plusieurs marqueurs d'authenticité n'indiquaient pas durant toute la durée du film qu'il s'agit d'une histoire vécue.

Néanmoins, cette séquence qui montre Erik Lapied grimpant pour faire des repérages est mise en scène, prévue et écrite avant d'être tournée. Elle vient poser les bases de ce que sera le film: l'aventure d'une femme et d'un homme qui doivent vivre avec la montagne pour accomplir leur travail: filmer la vie sauvage. L'écriture filmique de cette deuxième séquence de l'introduction est dramatisée. Il n'est pas nécessaire pour observer les bouquetins de passer par cette paroi et d'effectuer cette escalade. Pour autant, il est nécessaire pour la dramatisation du film que le personnage montre sa volonté ainsi que les risques qu'il prend pour vivre avec, et de, sa passion : la montagne et la vie sauvage.

Dans le film de Jean-Michel Bertrand, comme dans celui d'Anne et Erik Lapied, l'ajout de l'humain constitue une seconde ligne narrative parallèle et augmente l'intensité dramatique. Dans un rapport documentaire avec le spectateur, les réalisateurs témoignent de leurs expériences. Cependant, la réalité recréée pour les besoins du film n'est pas neutre. Les cinéastes animaliers se filment à l'extérieur, au milieu de la nature et quand ils le peuvent, proches des animaux. Ils sont parfois filmés comme les animaux : en plan large au sein de leur milieu de vie, dans leur besogne quotidienne. Être avec les animaux, dans la nature, c'est démontrer une connaissance des milieux et les savoir-faire techniques audiovisuels et naturalistes. Cette connaissance est nécessaire pour savoir où filmer les animaux et adapter les techniques de prises de vues aux milieux naturels. De plus, elle permet d'optimiser le tournage en fonction des contraintes et d'agir de façon à ne pas perturber la faune.

Dans ces images des cinéastes animaliers au travail, on regarde vivre le cinéaste, comme lui regarde vivre les animaux. Il est adapté à son milieu, il vit à l'extérieur et il connait bien le terrain. Il le dit et le montre. Ce métier n'est pas présenté comme une contrainte ou un devoir, mais comme un plaisir et une vocation.

\section{La construction de l'image du cinéaste animalier : de l'aventurier à la vocation}

15 En se filmant, en se mettant en scène, les cinéastes produisent des représentations assez stéréotypées de leurs activités et contribuent à poser les bases d'une identité professionnelle commune. Nous avons identifié cinq grands éléments qui participent à la construction de l'image du cinéaste animalier.

16 Premièrement, presque toutes les images des cinéastes animaliers dans leur activité sont consacrées à les montrer avec ou derrière leurs caméras. La caméra est l'outil principal du cinéaste, elle devient un prolongement de son corps. Partout où l'on suit un cinéaste, on suit également une caméra, le plus souvent juchée sur un pied ${ }^{10}$. Le cinéaste animalier a longtemps croulé sous le poids d'un matériel très lourd. Son principal travail physique est de porter la caméra dans toutes les conditions météorologiques et sur tous les terrains. Au tout début du film $A u$ cœur du tournage (Frédéric Chaudier, 2015), le réalisateur et producteur ${ }^{11}$, Frédéric Fougea, est mis en scène dans la nature, en train de porter du matériel lourd de tournage. Ce trépied qu'il transporte, non sans peine, constitue l'objet symbolique des cinéastes animaliers. La difficulté de l'exercice physique et sa mise en scène font écho à la domination 
masculine régnant dans le milieu de l'audiovisuel et dont le milieu des cinéastes animaliers n'est pas exclu ${ }^{12}$.

Le cinéaste est souvent représenté seul. Avec ou sans caméra, la solitude est présentée comme un attribut du cinéaste animalier, pour le repérage ou pour le tournage. Dans Sur les traces du renard (Jérôme Bouvier, 2007), l'un des cinéastes, Cyril Barbançon, part seul, un soir, avec le renard. Il ne revient que le lendemain matin, heureux d'avoir été accepté par l'animal qui lui a offert un instant magique. Cette escapade solitaire est traitée comme une épreuve initiatique: après l'avoir franchie, les cinéastes sont acceptés définitivement par le renard. Ils vont pouvoir le suivre à leur guise. Même Anne et Erik Lapied, dont le travail de cinéaste animalier se conjugue avec leur vie de couple, partent toujours chacun de leur côté dans la montagne. Parce que «la solitude affine les sens, les aiguise. Elle nous rend plus attentif, plus discret. Seul, à bon vent, nous approchons mieux l'animal farouche. Nous augmentons les chances d'être là, d'être là au bon moment pour observer ce qui s'offre à nous. $»^{13}$

La connaissance approfondie voire intime de la nature constitue une troisième caractéristique des cinéastes animaliers, qui démontrent ainsi l'un de leurs principaux atouts. Laurent Charbonnier, qui a réalisé entre autres Les Animaux amoureux (2007) pour le cinéma, est le personnage principal d'un film documentaire produit par sa propre société et réalisé par la fille de sa compagne, Mathilde Louveau. Ce film se nomme Chasseur d'image (2014): une façon pour le réalisateur de se placer dans une relation particulière de l'homme face à la nature. Le travail du cinéaste animalier est un travail de "chasseur d'images »; ainsi son objectif n'est pas de s'approprier le corps de l'animal en le tuant, mais bien de capter l'image en mouvement de l'être vivant. Car ses mouvements et ses comportements permettent de capter son âme. Ainsi c'est l'image animée, cinématographique ou vidéo qui permet de la capter ${ }^{14}$. Le chasseur " d'images animées de l'animal » possède une dimension mystique, qui le distingue du chasseur. Il ne faut pas tuer l'âme de l'animal, lui enlever la vie, mais la capturer à travers l'objectif de la caméra. La dimension mystique de cette tâche apparaît de différentes façons, mais elle est toujours présente dans les représentations du travail du cinéaste animalier et intégrée dans leurs réflexions :

« Donc t'y vas dans un but, t'es en train d'effectivement... y a un chamois devant toi, et puis tu sais pas pourquoi à un moment tu penses écureuil et tu te retournes puis il y a un écureuil derrière. Mais tu l'avais pas vu, mais tu le sens. C'est arrivé quand même plusieurs fois comme ça. Ça je trouve que c'est des choses qui se développent au fil des années. Alors après au niveau cinéma...c'est une sensibilité qui...comment filmer l'invisible? Tu vois ça se dit pas ça...tu le dis pas dans le film...mais je pense que ça doit se sentir. » (Homme, réalisateur, opérateur de prises de vue, 60 ans) ${ }^{15}$

La quatrième caractéristique de la figure du cinéaste animalier est sa proximité avec la vie sauvage. Ce sont des images d'hommes qui s'enfoncent dans la nature et qui vont devoir vivre et survivre avec elle. Dans un deuxième extrait de Vertige d'une rencontre, Jean-Michel Bertrand monte dans la montagne avec son âne. Par la suite, le cinéaste est obligé d'abandonner son compagnon car il parvient dans un endroit dangereux, rempli de failles. L'aventurier qu'il est, doit sauter par-dessus des ouvertures inquiétantes parmi les rochers. Enfin, il pénètre dans une grotte présentée comme son lieu de vie pour les prochains jours. Dans cette séquence et durant tout le film le réalisateur est mis en scène, toujours seul, comme un ermite. Ces figures de l'homme solitaire face à la nature valorisent leur passion pour la nature et leur vocation pour ce travail. L'aspect vocationnel s'exprime notamment à travers la dissociation du métier d'avec les 
nécessités matérielles et à travers une croyance très souvent affichée : celle d'avoir été conduit naturellement, par envie et par goût, à cette activité ${ }^{16}$.

3. Images extraites des films Vertige d'une rencontre, Sur les traces du renard et Voyage au bout de I'hiver.
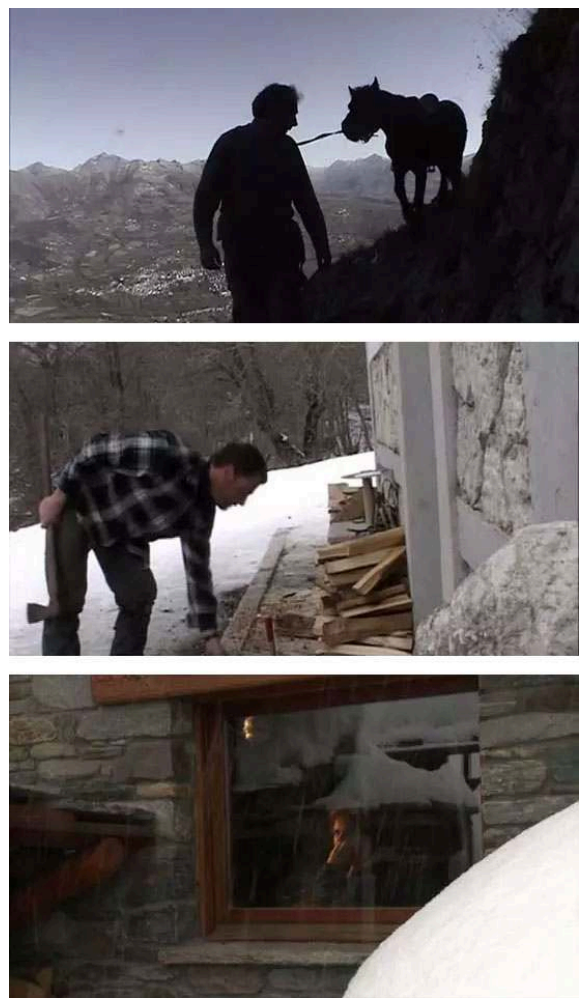

Le cinéaste animalier solitaire, dans un quotidien où la nature et les éléments dictent ses conditions de vie.

Dans les cas que nous venons de présenter, le cinéaste est loin de chez lui. Mais il peut aussi tourner dans la nature proche, montrant alors la cinquième caractéristique du métier : un dévouement complet pour son travail. Dans le making-of de Microcosmos, une séquence débute avec la localisation du tournage et plus particulièrement du studio de tournage : une maison dans l'Aveyron, aux portes de la nature. Il s'agit de la maison des deux réalisateurs, Claude Nuridsany et Marie Pérennou. La caméra pénètre dans la maison, dans le studio équipé d'un matériel technique lourd et encombrant sur lequel le cinéaste travaille. Il manipule des objectifs, puis présente un système complexe inventé pour l'occasion. On voit ensuite un petit bout de nature dans ce studio: le décor devant lequel se promène la caméra téléguidée. Le plateau de tournage, et donc le lieu de travail, se situe au sein de la sphère privée.

Pour Erik et Anne Lapied, dans Voyage au bout de l'hiver, être chez soi signifie continuer à travailler. Ils se filment devant leur ordinateur alors qu'ils regardent leurs images, ou devant une feuille de papier pendant l'écriture du commentaire. Erik Lapied installe même des projecteurs afin d'éclairer la fouine qui tourne autour de leur maison la nuit et pouvoir la filmer. Il n'y a pas de séparation entre vie privée et travail. Et si elle existe, c'est pour mieux nier l'existence d'une vie personnelle. Jean-Michel Bertrand dans Vertige d'une rencontre, part plusieurs jours en quête de l'aigle royal, dans des conditions parfois difficiles. Jamais il ne sera fait référence à sa vie dans la vallée. Toutes les 
séquences dans lesquelles il apparaît se déroulent en montagne, sur son lieu de travail. Dans Sur les traces du renard, le spectateur partage le quotidien pendant des mois, de l'hiver à l'été, de trois cinéastes animaliers qui vivent dans un petit gîte rustique et peu équipé. Cinq mois entiers semble-t-il, sans coupure, sans retour à leur famille.

Equipé de son matériel de tournage, seul avec sa grande connaissance du milieu naturel qui lui permet d'être proche de la vie sauvage et très dévoué à son travail, la représentation de l'idéal-type des cinéastes animaliers apparaît ainsi dans les images qu'ils produisent de leur travail.

\section{La construction de l'image du cinéaste animalier : de l'artiste à l'artisan}

Le métier est présenté comme une vocation et à l'image cela se traduit par l'exposition de tous les bénéfices que procure le travail du cinéaste animalier. Comme dans les professions artistiques, il existe dans celle des cinéastes animaliers des revenus nonmonétaires, ce que Pierre-Michel Menger nomme « des gratifications psychologiques et sociales, [des] conditions de travail attrayantes, [et une] faible routinisation des tâches, etc. $\gg^{17}$ Cinéaste animalier est un choix de vie présentant beaucoup de contraintes mais également de nombreux avantages, souvent très valorisés par les professionnels : une grande autonomie, la vie dans la nature, le lien avec les animaux. Et ce sont ces gratifications qui sont exposées dans les images que les cinéastes proposent d'euxmêmes.

Dans une séquence de Vertiges d'une rencontre, de Jean-Michel Bertrand, le cinéaste parcourt la montagne en skis de randonnée. Des images qui, sans le son, seraient plus associées aux loisirs, aux vacances, aux plaisirs de la montagne. Il traverse des paysages magnifiques et, parvenu au sommet de la montagne, il commence une descente à ski. Un parallèle est fait avec des chamois qui jouent sur un névé. Le rapprochement entre le cinéaste et les animaux est une nouvelle fois mis en scène. Ici, deux gratifications non-monétaires évidentes apparaissent. Premièrement, le métier de cinéaste animalier implique une pratique sportive, dans un cadre naturel splendide ; son travail est associé à une activité de loisir en pleine nature. Deuxièmement, l'homme se rapproche des animaux sauvages, il vit proche d'eux, voire avec eux.

Troisième gratification sous-jacente : l'autonomie d'organisation. Le cinéaste animalier a besoin de temps; parfois de beaucoup de temps pour s'imprégner de la nature, ou à l'inverse imprégner la nature ${ }^{18}$. Ce temps, abondant, lui permet une certaine liberté. La seule contrainte temporelle qui peut lui être infligée le sera par le comportement des animaux ou le déroulement des saisons. Par exemple Jean-Michel Bertrand doit attendre un an supplémentaire car l'aigle qu'il voulait filmer a choisi une aire de reproduction qu'il ne peut atteindre. Dans Sur les traces du renard, Jérôme Bouvier et son équipe prennent cinq mois pour apprendre et connaître la nature qui les entoure, l'important est que le renard soit prêt à tourner au printemps.

Les cinéastes animaliers sont des professionnels qui peuvent se mettre en scène, ils utilisent leur image pour élaborer des histoires qui valorisent leur métier : une activité exigeante présentée comme une vocation, nécessitant des savoir-faire de passionnés se pratiquant à plein temps et fournissant des gratifications non-monétaires très fortes. 
Ces aspects font penser à une activité de production artisanale. En effet, comme l'a montré Yann Daré, le monde professionnel du cinéma et de l'audiovisuel possède une division du travail qui n'est pas de type industriel mais davantage de type artisanal ${ }^{19}$. L'artisanat est revendiqué par l'ensemble des cinéastes quelles que soient les conditions de travail dans lesquelles ils exercent. Les cinéastes qui travaillent dans des productions importantes se situent dans des industries divisées en métiers où chaque corporation use de son savoir-faire pour produire l'œuvre. La division du travail n'est pas perçue comme la marque d'une production industrielle mais comme l'addition successive de compétences d'artisans. Les cinéastes qui travaillent pour des productions aux moyens plus limités doivent effectuer une plus grande diversité de tâches, ainsi ils revendiquent un savoir-faire qui englobe l'ensemble de la chaîne de réalisation d'un film et mettent en avant leur autonomie et la modestie de leurs projets. L'idée d'un travail organisé comme l'artisanat apparaît attractive pour tous: la production en petite équipe, dans laquelle chacun effectue une tâche qui correspond à un savoir-faire (précis ou global), permet de gommer la division du travail et la hiérarchisation des rapports sociaux entre décideurs et exécutants. Sous-entendu, l'égalité s'établirait de ce fait dans les rapports sociaux au sein d'une petite équipe d'artisans responsable de la production d'une œuvre et entre les différents cinéastes artisans du champ de la production audiovisuelle. Cette perception de l'activité, due aussi à son fractionnement par projets successifs se retrouve dans les images que les cinéastes produisent d'eux-mêmes : utiliser des outils spécifiques, avoir un savoir-faire particulier et se montrer seul ou en équipe restreinte avec une grande liberté d'organisation.

\section{Les éléments de construction de l'image}

A côté des savoir-faire, compétences, pratiques et savoir-vivre valorisés par les cinéastes animaliers, d'autres aspects du métier, pourtant importants sont laissés dans l'ombre. Les cinéastes ont fait des choix. Par exemple, Erik et Anne Lapied au début de Voyage au bout de l'hiver, mettent en scène les conditions incroyables du travail de cinéaste de montagne : une tempête de neige. Ils ne commencent pas par montrer les heures de travail que représentent le dérushage ${ }^{20}$ et le montage du film.

Un film se crée en trois étapes: la préparation (écriture du scénario, repérage, programmation et répartition du travail), le tournage, et bien sûr, la post-production (le dérushage, le montage et le mixage). Les deux étapes qui encadrent le tournage sont très peu filmées et présentées dans les films auxquels nous nous intéressons. Nous apercevons à de rares occasions le travail d'écriture et de préparation du tournage. Dans le making-of du Peuple Migrateur, on nous montre deux fois brièvement Jacques Perrin entouré de son équipe autour d'une table, en train de discuter. L'essentiel du film se concentre sur l'élevage des animaux et le tournage avec eux. Rien sur le montage ou la création de la musique. Le making-of de Microcosmos ne présente pas la phase de développement de l'outil de tournage : cette machine très complexe qui est pourtant au centre du film. Dans Vertige d'une rencontre, Jean-Michel Bertrand est déjà en train de tourner dès le premier plan, rien de la préparation et rien du montage ne seront montrés. Voyage au bout de l'hiver ne laisse place qu'à une seule scène de dérushage des images, devant lesquels Anne et Erik Lapied discutent, sans qu'on puisse entendre ce qu'ils se disent. Ce plan ne dure que quelques secondes. 
Les Lapied, cinéastes animaliers qui recherchent l'autonomie, ont acquis une indépendance financière. Ils ne travaillent plus pour des productions télévisées et répondent peu à des commandes institutionnelles. Le couple se rémunère grâce à un système de projections itinérantes payantes au sein de leur région et par la vente de DVD. L'essentiel de leurs revenus provient de l'exploitation qu'ils font eux-mêmes de leurs films. Ce travail représente une énorme quantité de temps, puisqu'il faut organiser les tournées, faire l'affichage au préalable, transporter le matériel, installer les salles, animer la projection puis gérer la partie financière. Cette activité s'étale toute l'année avec un pic pendant les saisons touristiques. Or dans Voyage au bout de l'hiver, une courte séquence présente Erik en train d'accomplir une tournée de projection. Mais elle dure à peine 40 secondes. Dès qu'elle se termine Erik est déjà en train de grimper sur sa montagne. Cette séquence est montée sur une musique de guitare qui, associée aux plans filmés depuis l'intérieur de la voiture, fait penser à un road-trip. Le montage alterne deux fois les plans de la voiture et les plans du cinéaste en train de parler au public, avec entre deux, une brève interview dans laquelle il dit qu'il prend plaisir à regarder plusieurs fois son film. Le montage, en 40 secondes essaye de nous donner l'impression que la tournée dure longtemps, que les projections sont répétitives, mais le sujet est rapidement traité. Les Lapied ne travaillent pas non plus complètement seuls, contrairement à ce que pourrait laisser penser le film. Le générique l'indique : ils travaillent avec leur fille Véronique. Elle n'apparait jamais dans le film et il n'y est jamais fait allusion. Les Lapied préfèrent se montrer comme un couple de deux solitaires, qui ne se retrouvent que dans leur maison. Alors même que les images d'Erik qui grimpe sur le rocher ou d'Anne qui filme sous la neige, ont été faites par l'autre. S'ils sont parfois deux sur la montagne, ils ne le sont jamais à l'écran. 
4. Images extraites du film Voyage au bout de l'hiver.
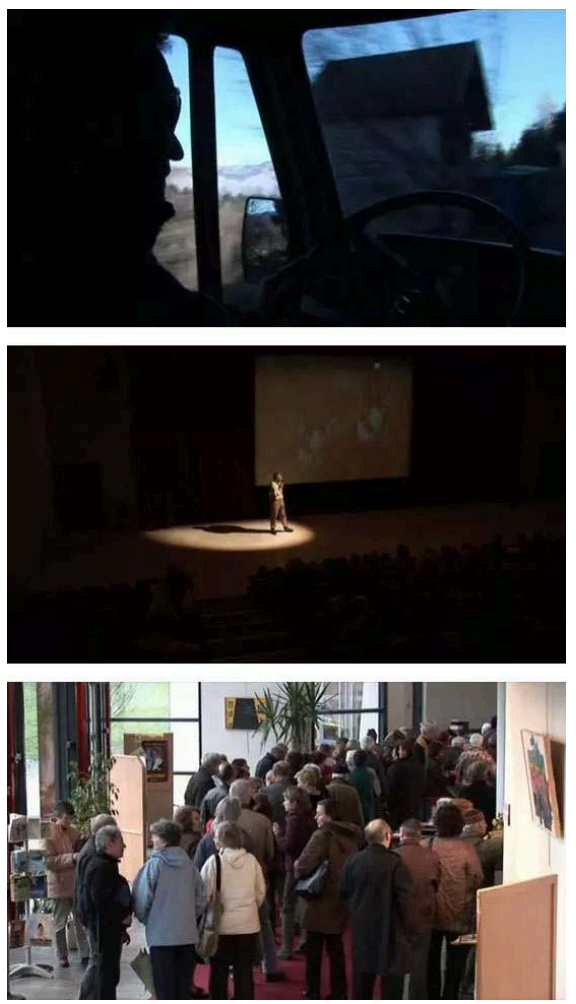

En quelques plans et seulement 40 secondes, l'autre travail des cinéastes-conférenciers Anne et Erik Lapied est décrit succinctement. On y distingue les déplacements nécessaires, le rôle de conférencier que le cinéaste doit occuper et le public qui entre dans la salle.

31 Il en est de même pour Vertige d'une rencontre, dans lequel Jean-Michel Bertrand est souvent filmé par une deuxième personne qu'on ne voit jamais. Même s'il a pu se filmer seul parfois, grâce notamment à sa petite caméra, il arrive que des plans en mouvement le suivent dans ses déplacements. Dans Sur les traces du renard, rien n'est visible de l'écriture ou du montage non plus. Les recherches du lieu de tournage sont présentées brièvement par Jérôme Bouvier au début du film et le scénario du Renard et l'enfant, le film pour lequel les cinéastes travaillent apparaît rapidement, avec la présentation du storyboard. Mais nous ne verrons rien d'autre que le tournage, comme si le travail du cinéaste animalier était exclusivement d'être sur le terrain, avec la caméra et les animaux. L'image donnée par les cinéastes animaliers dans les films qui les montrent dans leur activité est incomplète par rapport à la réalité de la pratique.

Les cinéastes animaliers qui développent leurs propres projets se trouvent dans la situation de devoir écrire le film. L'écriture est nécessaire voire indispensable dans certains contextes. Pour proposer un projet à un producteur ou un diffuseur, ou encore répondre à un appel d'offres d'un financeur institutionnel, il est indispensable au cinéaste de rédiger un scénario. C'est d'autant plus vrai que les exigences dans ce domaine ont augmenté. Les cinéastes animaliers qui ont commencé à travailler dans les années 1980 constatent qu'il est demandé de plus en plus d'écriture pour vendre des projets. C'est à eux auteurs-réalisateurs d'assumer le développement, afin, dans un premier temps, de convaincre des partenaires de le financer et dans un deuxième temps de préparer le tournage. En entretien, la question de leurs luttes économiques pour financer leurs activités revient régulièrement. Beaucoup sont perpétuellement en 
quête de financements ${ }^{21}$. Certains font face aux chaînes de télévisions dont les desiderata en matière de contenu éditorial et de forme documentaire doivent être respectés si on souhaite travailler avec elles. Les mêmes doivent ensuite s'associer avec des sociétés de production et négocier avec elles les conditions de tournage. Lorsqu'ils sont dans d'autres circuits économiques, les cinéastes animaliers effectuent des recherches de commandes d'institutions ou des projections itinérantes.

Dans les images qui les montrent au travail, rien de cet aspect de leur profession n'est exposé. Ils sont tous des professionnels autonomes qui n'ont pas à lutter pour le rester. Ils sont dans l'action du tournage. Aucun lien avec les producteurs et presque rien sur le travail d'écriture et de montage. Ils n'apparaissent pas en tant qu'auteur ou en tant que chef d'une équipe artistique. Ils se montrent avant tout comme des techniciens et des naturalistes. Les images réflexives idéalisent ainsi leur position d'artisans autonomes, réduisent l'activité du cinéaste à sa seule activité de tournage et simplifient celle-ci.

L'analyse des images que ces travailleurs de l'audiovisuel produisent sur eux-mêmes, nous permet ainsi de repérer les représentations similaires. Ainsi dans les making-of ou dans leurs films, les cinéastes animaliers illustrent et font perdurer le modèle idéal de leur profession. Ils construisent ensemble l'image que projette la profession au monde extérieur : autonomie du cinéaste qui est avant tout un chasseur d'image, respect des animaux qui nécessite la connaissance du milieu naturel et engagement total du professionnel dans son travail. La mise en scène de ce qu'ils considèrent comme le cœur de leur métier, la prise de vues, leur permet de le valoriser à travers l'image de l'aventure humaine et le don de soi. Artistes, de par la liberté et les gratifications nonmonétaires que leur procure leur pratique, ils s'affichent aussi comme des artisans dans la maîtrise des savoir-faire spécifiques au métier et la dimension créative de leur activité. Alors qu'ils cherchent à transmettre la réalité d'un monde sauvage duquel leurs contemporains ont tendance à s'éloigner, les cinéastes animaliers n'hésitent pas à s'affranchir de la réalité lorsqu'il s'agit de se mettre en scène soi-même. Ce sont les mêmes procédés qu'ils reproduisent quel que soit leur statut : producteurs, salariés de société de production ou auto-producteur de leur propre film. Tous, malgré les variabilités des contextes dans lesquels ils évoluent participent à reproduire des représentations communes de leurs métiers et à construire l'idée d'une expérience partagée. En analysant ces films, nous faisons apparaître une part de la culture d'une activité professionnelle. Ainsi l'étude de l'image dans notre recherche met en évidence et apporte un élément concret dans la démarche sociologique qui consiste à interroger l'élaboration d'un groupe professionnel dans un secteur d'activité particulier. 


\section{Bibliographie}

Blanc M. (2008) L'image-relation, sociologie du monde du cinéma documentaire (thèse), Université de Strasbourg.

$\mathrm{CNC}$, service des études (2003) Le contenu des DVD, rapport, 30p.

CNC service des études (2014) La place des femmes dans l'industrie cinématographique et audiovisuelle, rapport, 76p.

Danard B. \& Le Champion R. (2005), Les programmes audiovisuels, Paris, La Découverte.

Darre Y. (2006) « Esquisse d'une sociologie du cinéma », Actes de la recherche en sciences sociales, $\mathrm{n}^{\circ} 161-162$.

Horak J-C. (2006) «Wildlife documentaries: From Classical Forms to Reality TV », Film History, vol. $18, \mathrm{~N}^{\circ} .4$, Documentary before Verité pp. 459-475.

Krief J-P. (2006) « Journal d'un artisan producteur d'images », Communications, $\mathrm{n}^{\circ} 80$.

Laulan A-M. (1976), « Un film et ses filtres culturels », Communication et langages, $\mathrm{n}^{\circ}$ 30,. pp. 83-96.

Menger P-M. (2009), Le travail créateur, s’accomplir dans l'incertain, Lonray, Gallimard Seuil.

Naudier D. \& Rollet B. (Dir.) (2008) Genre et légitimité culturelle, Paris, L'Harmattan.

Péquignot B. (2006) « De l'usage des images en sciences sociales », Communications, $\mathrm{n}^{\circ} 80$.

Pinto A. (2011) « L'économie de la création, le financement du film documentaire », in Revue de synthèse, « caméra, terrain et sciences sociales ", $\mathrm{n}^{\circ} 3$.

Rot G. \& de Verdale L. (dir.) (2013) Le cinéma, travail et organisation, Paris, La dispute.

Sennet R. (2010), Ce que sait la main. La culture de l'artisanat, Paris, Albin Michel.

Sorignet P-E. (2010), Danser, enquête dans les coulisses d'une vocation, Paris, La découverte.

Zarca B. (1988) « Identité de métier et identité artisanale », Revue française de sociologie, 29-2.

\section{Filmographie}

Jean-Michel Bertrand (2010) Vertige d'une rencontre, documentaire, 75'.

Jérôme Bouvier (2007) Sur les Traces du renard, documentaire, 52'.

Jérôme Bouvier (2005) Ours polaire, avec ou sans glace, documentaire, 52'.

Jacques Cluzaud, Jacques Débats \& Jacques Perrin (2001) Le Peuple migrateur, Making-of 6'.

Pascal Gaubert (2011) Où notre regard ne porte plus, film d'école, 22'.

Romain Guénard (2012) Sur les rives de la Bohême, Film d'école, 24'.

Frédéric Labie (2013) On touche avec les yeux, Film d'école, 16'.

Anne \& Erik Lapied (2010) Voyage au bout de l'hiver, documentaire, 78'

Jérôme Lombard (2012) Jeux de piste, Film d'école, 23'

Mathilde Louveau (2014) Chasseur d'image, documentaire, 56'

Claude Nuridsany, Marie Pérennou (1996) Microcosmos, Making-of, 12' 


\section{NOTES}

1. Blanc Mathias, L'image-relation, sociologie du monde du cinéma documentaire (thèse), Université de Strasbourg, 2008

2. Si le genre auquel nous nous intéressons est en français qualifié d' " animalier ", il est communément catégorisé « wildlife » ("vie sauvage ») en anglais. Il est plus compliqué de filmer des animaux en totale liberté que de filmer des animaux habitués à la présence humaine et captifs. Ainsi, derrière la distinction sociale entre animaux sauvages et domestiques, se dissimulent deux pratiques différentes pour les cinéastes. Or nous nous intéressons à ceux qui développent une activité spécifique au monde sauvage.

3. Lamoureux Maxence, Les cinéastes animaliers en France, d'une diversité des productions et des parcours à l'émergence d'un groupe professionnel (thèse), Université de Poitiers, 2015

4. http://www.wild-touch.org/chronique-de-la-foret/, consulté le 15/11/2014

5. Le contenu des DVD, CNC, 2003, http://www.cnc.fr/web/fr/publications/-/ressources/19897

6. Citons par exemple Le Territoire des autres (François Bel, Michel Fano, Gérard Vienne, 1970), L'Arche et les déluges (François Bel, 1992), Les Animaux amoureux (Laurent Charbonnier, 2007)

7. La production audiovisuelle bénéficie française d'aides gérées par le Centre National de la Cinématographie et de l'image animée. La séparation des formes, documentaire et fiction, réalisée par le $\mathrm{CNC}$ pour organiser la répartition des financements contribue à structurer la production et la diffusion. Or les fictions animalières produites pour la télévision sont inexistantes en France.

8. Voir à ce sujet, entre autres : Où notre regard ne porte plus, Pascal Gaubert (2011) - Jeux de piste, Jérôme Lombard (2012), Sur les rives de la Bohême, Romain Guénard (2012) - On touche avec les yeux, Frédéric Labie (2013)

9. La désaturation consiste au moment du montage à diminuer l'intensité des couleurs, pour produire une image qui tire vers le noir et blanc.

10. Seul Christian Zuber, avec son émission justement nommée Caméra au poing, filmait les animaux à main levé.

11. Il n'est pas un technicien et en ce sens ne porte un trépied que pour la caméra qui le filme

12. Voire l'étude du CNC, La place des femmes dans l'industrie cinématographique et audiovisuelle, 2014, et l'ouvrage de Naudier Delphine, Rollet Brigitte (Dir.), Genre et légitimité culturelle, L'Harmattan, Paris, 2008

13. Anne Lapied, dans Voyage au bout de l'hiver d'Anne et Erik Lapied

14. Nous en revenons ici à l'étymologie même du terme " animal »: "être vivant mobile, doté du souffle vital ou anima», substantif dérivé de anima (âme.) ", Rey Alain (dir.), Dictionnaire historique de la langue française, Dictionnaires Le Robert, Paris, 1998, p.145

15. Propos recueilli dans le cadre du travail de recherche de l'auteur de l'article.

16. C'est ainsi que Pierre-Emmanuel Sorignet reconnaît aussi la dimension vocationnelle des danseurs professionnels dont il fait l'étude. Voir Sorignet Pierre-Emmanuel, Danser, enquête dans les coulisses d'une vocation, Editions de la découverte, Paris, 2010

17. Menger Pierre-Michel, Le travail créateur, s'accomplir dans l'incertain, Gallimard Seuil, Lonray, 2009, p.16

18. C'est à dire habituer les animaux sauvages à agir comme il le souhaite, en les nourrissant notamment.

19. Darre Yann, « Esquisse d'une sociologie du cinéma ", Actes de la recherche en sciences sociales, $\mathrm{n}^{\circ}$ 161-162, 2006, p.127

20. Le dérushage constitue la première étape de la post-production avant le montage. Il s'agit de visionner une première l'ensemble des images filmées (les rushs), de les classer selon leur qualité et leur sujet. Cette étape essentielle permet de gagner un temps précieux par la suite afin de retrouver rapidement les plans que l'on souhaite monter 
21. Voir l'article d'Aurélie Pinto, "L'économie de la création, le financement du film documentaire », in Revue de synthèse, « caméra, terrain et sciences sociales », n³, 2011

\section{RÉSUMÉS}

Les cinéastes au travail se filment parfois et proposent aux spectateurs de découvrir leur activité derrière la caméra. Dans les images du travail des cinéastes figurent des mises en scène et des montages qui décrivent et soulignent des caractéristiques particulières, comme des savoir-faire spécifiques, et proposent des représentations biaisées correspondant à l'idéal de l'activité. L'analyse proposée ici s'appuie sur un travail de terrain, d'observation et d'enquête réalisé dans le cadre d'une thèse portant sur la constitution de la profession de cinéaste animalier en France. Dans les making-of de leurs films ces travailleurs de l'audiovisuel élaborent l'image idéal de leur activité. L'étude de l'image est ainsi susceptible d'apporter un élément concret dans une démarche sociologique qui interroge l'élaboration d'un groupe professionnel.

Filmmakers at work sometimes film themselves and offer spectators an exploration of what goes on behind the camera. These pictures of filmmakers work feature staging and editing which describe and accentuate specific characteristics (such as specific know-how), and provide thwarted representations of the trade. Our analysis relies on fieldwork, observations and a survey conducted for a doctoral thesis on the constitution of the profession of wildlife filmmakers in France. In their making-of, these workers of the video design the ideal image of their trade. Studying image is then likely to bring a actual element in a sociological approach questioning the making of a professional group.

\section{INDEX}

Keywords : work, sociology, profession, trade, image, making-of, wildlife film, documentary, film, nature

Mots-clés : sociologie, profession, travail, image, making-of, cinéma, animalier, documentaire, film, nature

\section{AUTEUR}

\section{MAXENCE LAMOUREUX}

Maxence Lamoureux est docteur de l'Université de Poitiers, sa thèse consacrée à la sociologie du monde des cinéastes animaliers français, a été soutenue en septembre 2015. Il est par ailleurs, réalisateur de documentaire sociaux et nature.Communications :

"L'indépendance en question dans le groupe professionnel des cinéastes animaliers", Congrés de l'AFS 2013, RT1

"Jacques Perrin ou l'exigence qualitative et populaire. La démarche originale du réalisateur et producteur de films animaliers pour le cinéma", Colloque organisé par "Jacques Perrin, portrait d'un artiste engagé", Avril 2014, RIRRA 21, l'Université de Montpellier 3 
Docteur en sociologie

GRESCO

Université de Poitiers 